\title{
Orbital modulation of X-ray emission lines in Cygnus X-3
}

\author{
O. Vilhu ${ }^{1}$, P. Hakala ${ }^{2}$, D. C. Hannikainen ${ }^{1,4}$, M. McCollough ${ }^{3}$, and K. Koljonen ${ }^{3,1}$
}

\author{
1 Observatory,Tähtitorninmäki (PO Box 14), 00014 University of Helsinki, Finland \\ e-mail: osmi .vilhu@astro.helsinki.fi \\ 2 Tuorla Observatory, University of Turku, Väisäläntie 20, 21500 Piikkiö, Finland \\ e-mail: pahakala@astro.utu.fi \\ ${ }^{3}$ Harvard-Smithsonian Center for Astrophysics, 60 Garden Street, Cambridge, MA 02138, USA \\ e-mail: mccolml@head-cfa.harvard.edu \\ 4 Metsähovi Radio Observatory/TKK, Metsähovintie 114, 02540 Kylmälä, Finland \\ e-mail: diana@kurp.hut.fi
}

Received 5 November 2008 / Accepted 19 April 2009

\begin{abstract}
Aims. We address the problem where the X-ray emission lines are formed and investigate orbital dynamics using Chandra HETG observations, photoionizing calculations and numerical wind-particle simulations. The aims were to set constraints on the masses of the components of this close binary system consisting of a Wolf-Rayet (WR) star and a compact component and to investigate the nature of the latter (neutron star or black hole). The goal was also to investigate P Cygni signatures in line profiles.

Methods. The observed Si XIV (6.185 Å) and S XVI (4.733 Å) line profiles at four orbital phases were fitted with P Cygni-type profiles consisting of an emission and a blue-shifted absorption component. Numerical models were constructed using photoionizing calculations and particle simulations. In the models, the emission originates in the photoionized wind of the WR companion illuminated by a hybrid source: the X-ray radiation of the compact star and the photospheric EUV-radiation from the WR star.

Results. Spectral lines with moderate excitation (such as Si XIV and S XVI) arise in the photoionized wind. The emission component exhibits maximum blue-shift at phase 0.5 (when the compact star is in front), while the velocity of the absorption component is constant (around $-900 \mathrm{~km} \mathrm{~s}^{-1}$ ). Both components, like the continuum flux, have intensity maxima around phase 0.5 . The simulated Fe XXVI Ly $\alpha$ line (1.78 A, H-like) from the wind is weak compared to the observed one. We suggest that it originates in the vicinity of the compact star, with a maximum blue shift at phase 0.25 (compact star approaching). By combining the mass function derived with that from the infrared He I absorption (arising from the WR companion), we constrain the masses and the inclination of the system. Conclusions. The Si XIV and S XVI lines and their radial velocity curves can be understood in the framework of a photoionized wind involving a hybrid ionizer. Constraints on the compact star mass and orbital inclination $(i)$ are given using the mass functions derived from the Fe XXVI line and He I $2.06 \mu \mathrm{m}$ absorption. Both a neutron star at large inclination ( $i \geq 60$ degrees) and a black hole at small inclination are possible solutions. The radial velocity amplitude of the He II $2.09 \mu \mathrm{m}$ emission (formed in the X-ray shadow behind the WR star) suggests $i=30$ degrees, implying a possible compact star mass between 2.8-8.0 $M_{\odot}$. For $i=60$ degrees the same range is $1.0-3.2 M_{\odot}$.
\end{abstract}

Key words. black hole physics - accretion, accretion disks - stars: binaries: spectroscopic - stars: winds, outflows stars: individual: Cygnus X-3 - stars: neutron

\section{Introduction}

Cygnus X-3 (4U 2030+40, V1521 Cyg) is a high-mass X-ray binary (HMXB) located at a distance of $9 \mathrm{kpc}$ with a close binary orbit $(P=4.8$ h; Liu 2007, Hanson 2000). The compact star is either a neutron star or a black hole and the companion is a WN5-7 type Wolf-Rayet (WR) star (van Kerkwijk 1992, 1996), with strong evidence pointing to an early-type WN star (Fender et al. 1999). The system appears to be engulfed in an extended envelope produced by the wind of the WR star. This notion is supported by the fact that massive winds are generally observed in Wolf-Rayet stars (Langer 1989; Crowther 2007).

Specifically with regard to Cyg X-3, large and complex absorption is found in broadband X-ray spectral studies (e.g. Vilhu 2003; Hjalmarsdotter 2008a; Szostek \& Zdziarski 2008), the orbital modulation of X-ray light curves can be attributed to electron scattering absorption in an asymmetric wind (with respect to the X-ray emitter, Pringle 1974; Willingale 1985), and the source exhibits a very rich Chandra HETG emission line spectrum (Paerels 2000). The spectrum shows radiative recombination continuums (RRC) that is a clear indication of a photoionized plasma (an alternative to a collisionally ionized gas). Liedahl et al. (2000) and McCollough et al. (2000) report P Cygni profiles in some X-ray emission lines. Stark \& Saia (2003) found Doppler modulation of X-ray lines in Chandra data from April 2000 when Cyg X-3 was in the high state, and used this to constrain the compact star mass to below 3.6 solar masses.

In the present paper we analyse one long Chandra HETG observation taken during a high state of Cyg X-3. We fit the Si XIV $6.185 \AA$ A Ly $\alpha$-line at four orbital phases with a P Cygnitype profile (an emission component with a blue-shifted absorption component). Results for the S XVI Ly $\alpha$-line at $4.73 \AA$, the Fe Ly $\alpha$ line at $1.78 \AA$, and the $\mathrm{He} \alpha$ line at $1.859 \AA$ are also presented. Using a two-component hybrid ionizing flux arising from the compact object and the WR star and the XSTAR photoionizing code (Kallman 2006; for a review of photoionized winds see also Liedahl 2001), we estimate line emissivities in the 
radiation field. Radial velocities and lightcurves were computed from a wind model based on particle simulations in the binary potential and compared with those we observed. We conclude by discussing constraints on the orbital inclination, component masses, and wind velocity.

\section{Chandra observations}

Chandra High Energy Transmission Grating (HETG) observations (OBSID: 7268, PI: McCollough) were scheduled on 2006 January 25-26 as a part of a large international campaign to study Cyg X-3 during an active state. The observations started on MJD 53760.59458 (when the source was at phase 0.053) and went through to MJD 53761.42972 (phase 4.240). During these observations Cyg X-3 was in a high soft state (quenched radio state) with an average $R X T E / A S M$ count rate around 30 cps (corresponding to $400 \mathrm{mCrab}$; typical hard state count rates are less than $10 \mathrm{cps}$ ).

The HETG consists of two gratings, the Medium Energy Grating (MEG) and the High Energy Grating (HEG). The observations were done in CC mode (continuous clocking) with a window filter applied to the zeroth order. The CC mode was used to avoid pileup in the dispersed spectra and the window filter was applied to avoid telemetry problems that could result from excessive counts in the zeroth order. A measure of pileup is the count rate per frame time (time to read out the chip) in a three-by-three pixel island. For this data set this quantity was always less than 0.02, and from Poisson statistics this implies a pileup rate of less than 1 per cent. The downside of using this mode is that spatial information is lost and the background is an order of magnitude higher.

During spectral extraction regions, either side of the dispersed spectra were used to create a background spectrum to be used in the analysis. In addition to instrumental background components this background was also used in the removal of components due to scattering.

The observation lasted over four orbital cycles and was divided into 4 and 10 phase groups. For each group, HEG and MEG spectra with their responses were extracted and first-order spectra used in the present study. The spectral resolutions were $0.023 \AA$ and $0.012 \AA$ for MEG and HEG, respectively (corresponding to $1160 \mathrm{~km} \mathrm{~s}^{-1}$ and $580 \mathrm{~km} \mathrm{~s}^{-1}$ at the Si XIV line).

The spectra were analysed with XSPEC (version 11) and extracted into a format which could be used by programs written for IDL (version 6.2). We concentrated on the Si XIV $6.185 \AA$ $\mathrm{Ly}_{\alpha}$-line as it is free of blends and the line profiles should be reliable. However, profile fits for the S XVI Ly $\alpha$ line at $4.73 \AA$ are also included in this work as well as the radial velocity study of the iron lines at $1.78 \AA$ and $1.86 \AA$.

\section{XSTAR simulations}

XSTAR is a computer code that calculates radiation effects in a cloud surrounding an ionizing source (Kallman 2006); in particular, the complex emission line spectrum of different species is computed. The important input parameters are the chemical composition, density, and radius of the spherical cloud, and the $\mathrm{X}$-ray/EUV spectrum of the central source. Of particular relevance is the radiation (ionizing luminosity) above the hydrogen ionization limit of $13.6 \mathrm{eV}$. The code was run in constant density mode, and the temperature was calculated to be the equilibrium value. The input parameters are listed in Table 1 and explained below.
Table 1. The ionizing sources and gas parameters used in the XSTAR code.

\begin{tabular}{lcc}
\hline \hline Compact star & WR star & Gas envelope \\
\hline CompPS & BB & $N_{H}=5 \times 10^{23} \mathrm{~cm}^{-2}$ \\
$T_{\text {bb }}=0.71 \mathrm{keV}$ & $T_{\text {eff }}=0.01 \mathrm{keV}$ & $N=10^{11} \mathrm{~cm}^{-3}$ \\
$L=2.46 \times 10^{38} \mathrm{erg} / \mathrm{s}$ & $L=5 \times 10^{38} \mathrm{erg} / \mathrm{s}$ & $\mathrm{H}=0.1, \mathrm{He}=10, \mathrm{CNO}$ \\
\hline
\end{tabular}

Since the source of the wind is a WN(4-7) star (van Kerkwijk et al. 1996) we used helium-rich gas abundances with $\mathrm{CNO}$ equilibrium values: $\mathrm{H}=0.1, \mathrm{He}=10, \mathrm{C}=0.56$, $\mathrm{N}=40, \mathrm{O}=0.27$; the other elements were set to solar, i.e. 1 (Crowther 2007; Vilhu 2007). The role of abundances is not significant, however, since we are not concerned with absolute values of fluxes, abundances or densities. The column $\left(N_{H}=\right.$ $\left.5 \times 10^{23} \mathrm{~cm}^{-2}\right)$ and particle density $\left(N=10^{11} \mathrm{~cm}^{-3}\right)$ were chosen resulting in a cloud of size $\sim 1 \mathrm{AU}$. The $N_{H}$-value is compatible with the Thomson scattering origin of the orbital modulation of the hard X-ray light curves (Vilhu et al. 2007).

Both binary components were used as the ionizing radiators, thus forming a hybrid ionizer. To obtain initial estimates for the parameters of the X-ray ionizing source (i.e. the compact star), we fit $R X T E / P C A$ spectra (xp5100, xp5200, xp5300) that were simultaneous to the Chandra observation in XSPEC with a Comptonizing model (compPS, Poutanen \& Svensson 1996) attenuated by photoelectric absorption (wabs). For a distance of $9 \mathrm{kpc}$, the resulting unabsorbed bolometric luminosity is $2.46 \times 10^{38} \mathrm{erg} / \mathrm{s}$. The model includes photoelectric absorption $\left(8 \times 10^{22} \mathrm{~cm}^{-2}\right)$, a black body radiator $\left(T_{\mathrm{bb}}=0.71 \mathrm{keV}\right)$, a Comptonized component (with $k T_{\mathrm{e}}=5.8 \mathrm{keV}$ and $\tau=2.0$ ) and a reflection component (with relative reflection $=4.5$ ). The spectrum is soft and dominated by a disc black body, typical for X-ray binaries in the high state. Note that we do not claim that this model is the best one to represent Cyg X-3 in the high state - the true absorption is probably more complex than is assumed here and the compPS model too simple. However, the model does give a viable numerical framework for our present purposes, including a correct value for the ionizing luminosity.

For the WR star we used values similar to those of V444 Cygni, which is a WN5 + O6 eclipsing binary: a mass of $10 M_{\odot}$, a radius of $0.92 R_{\odot}$, a luminosity of $10^{5.1} L_{\odot}$ and $T_{\text {eff }}=$ $115000 \mathrm{~K}$ (Langer 1989). We approximate the spectrum with a black body of $T_{\text {eff }}=0.01 \mathrm{keV}(116000 \mathrm{~K})$ and bolometric luminosity of $5 \times 10^{38} \mathrm{erg} / \mathrm{s}$; the ionizing luminosity is then $4.47 \times 10^{38} \mathrm{erg} / \mathrm{s}$. The incident and outgoing fluxes are illustrated in Figs. 1 and 2. The outgoing flux was reddened by $A_{V}=20$ and $A_{V} / N_{H}=5.3 \times 10^{-22} \mathrm{~cm}^{2}$, following Tables 21.6 and 21.7 in Cox (2000).

Figures 1 and 2 show that even though the model outlined above adequately fits the observed Chandra HEG spectrum, it does not fully reproduce the infrared (IR) continuum and there is a soft X-ray excess visible relative to the continuum. Depending on whether Cyg X-3 is in quiescence or in outburst, the infrared magnitudes can vary by a magnitude (Hanson et al. 2000). Figure 1 shows the infrared magnitudes given in Hanson et al. (2000): $m_{H}=13.1 \pm 0.1$ and $m_{K}=11.7 \pm 0.1$, based on observations during quiescence by Fender et al. (1996). The soft $\mathrm{X}$-ray excess is likely due to background issues and/or an inadequate representation of the absorption in the RXTE/PCA spectra. However, these discrepancies do not affect the results presented here.

XSTAR requires as input a single ionizing source in the cloud center, although in our case we have two distinct 


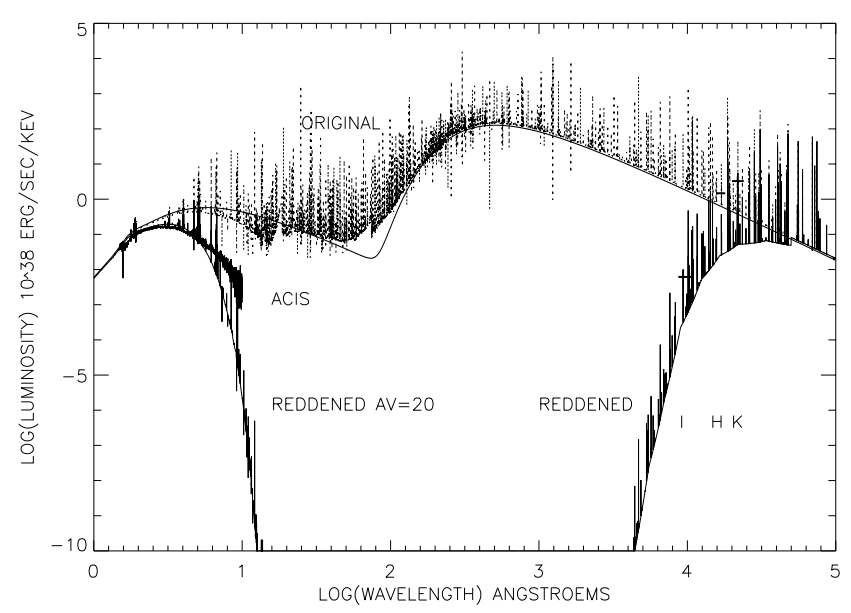

Fig. 1. The outgoing broadband spectrum. The dotted line traces the original spectrum and the solid line the reddened spectrum as computed with the hybrid ionizer (see the text and Table 1). The observed IR magnitudes (Hanson et al. 2000, short horizontal lines) and the mean Chandra HETG spectrum (OBSID 7268) are shown. The solid continuous line is the spectrum of the incident hybrid source.

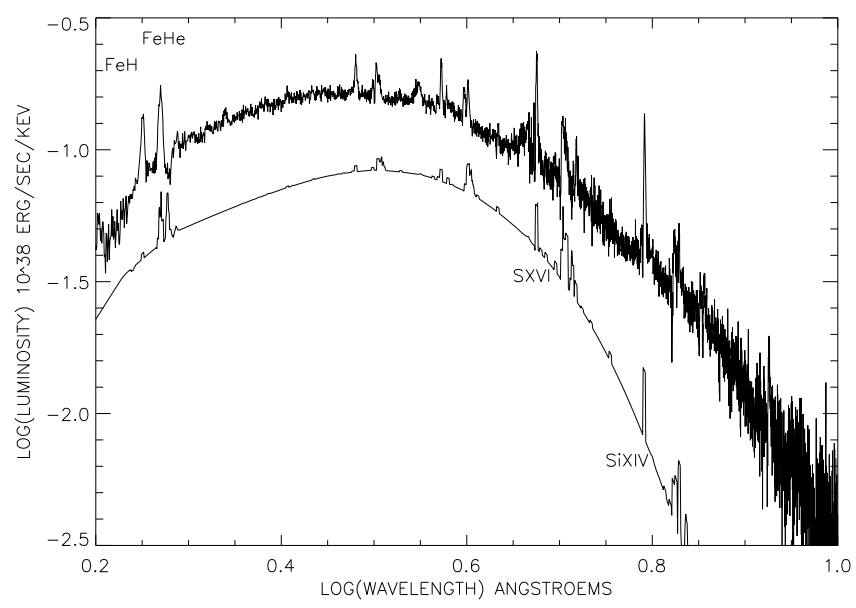

Fig. 2. A zoom of the simulation of Fig. 1 (shifted vertically by -0.4 ) overplotted with the mean Chandra HETG-spectrum (OBSID 7268).

ionizers. Hence, several runs with different He-star luminosities and distances from the compact object were performed (using spherical shells) so as to interpolate the correct radiation field at any spatial point where the line emissivity was computed (see Fig. 3 for Si XIV).

Additional emissivity grids were computed by varying the particle density. The degree of photoionization is governed by the density of the gas through the the ionization parameter, which is proportional to the ionizing luminosity and inversely proportional to distance squared and particle density, $\xi=L /\left(n R^{2}\right)$. Ionization is sensitive to the density of the gas (which itself depends on the ionizing species in question): it can increase or decrease in accordance. The final line emission was then computed in the wind model by fixing the mean density to $10^{11} \mathrm{~cm}^{-3}$ (see Fig. 4 for Si XIV). This mean density gives the correct electron scattering opacity along the line of sight and produces a partial eclipse in the light curve by a factor of two, as observed. A similar procedure was undertaken to estimate the absorption in the transmitted spectrum.

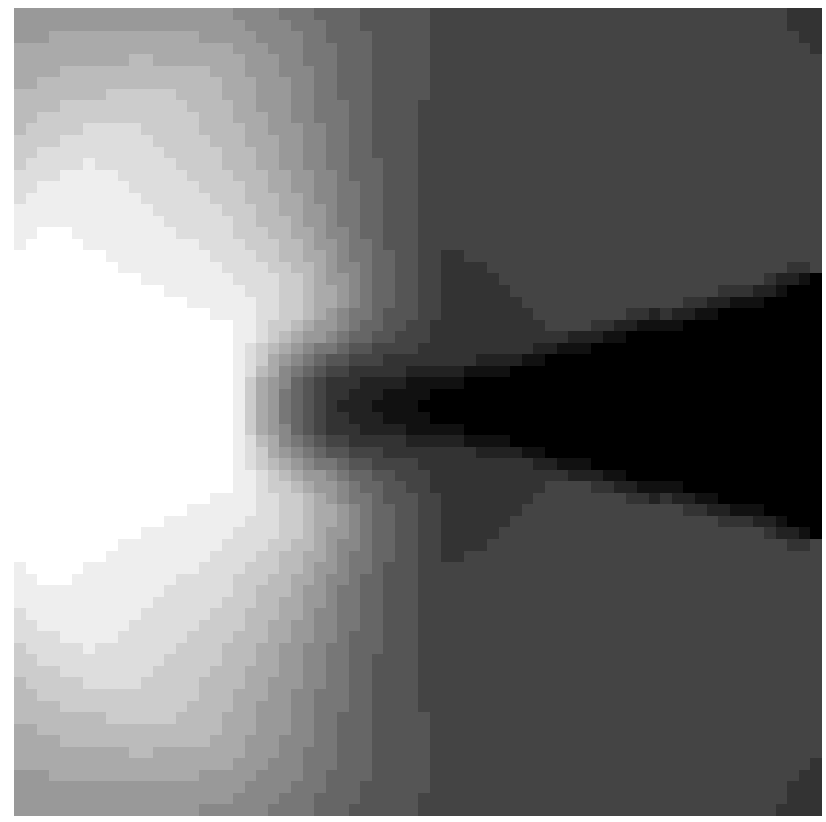

Fig. 3. Si XIV Ly $\alpha$-line emissivity per unit volume for constant density $N=10^{11} \mathrm{~cm}^{-2}$ (on orbital plane) based on the hybrid ionizer (Table 1). The plot size is $32 R_{\odot}$, which corresponds to ten binary separations. The $\mathrm{X}$-ray shadow of the WR-companion is seen on the right. The compact component is in the dark spot slightly to the left of figure center. In this grey scale white corresponds to maximum and black to zero emissivity. At orbital phases 0.0 and 0.5 the system is viewed from the right and left, respectively.

The emission scales relative to the equilibrium temperature as $T^{-0.6}$, which explains the enhanced emission when the extra heater, the WR star, is farther away (see Fig. 3). The line absorption in the transmitted flux, in turn, has no temperature dependence.

\section{Particle simulations}

For modeling the velocity and density fields so as to obtain a reasonable input model for XSTAR, test particles were injected symmetrically from the Wolf-Rayet companion and their trajectories were followed in the binary potential. Neither acceleration nor interaction between the particles was assumed, which is a good assumption for the supersonic velocity of the wind. Typical values for the wind velocity and mass loss of WR stars are $2000 \mathrm{~km} \mathrm{~s}^{-1}$ (observed wind velocities lie in the range $1000-3000 \mathrm{~km} \mathrm{~s}^{-1}$ (Cox 2000)) and $3 \times 10^{-5} M_{\odot} /$ year, respectively (Langer 1989). The masses of WN stars are between $10-80 M_{\odot}$, while the range of WC stars is narrower $\left(9-16 M_{\odot}\right.$, Crowther 2007).

In our simulations we used wind velocities between 1200-2200 $\mathrm{km} \mathrm{s}^{-1}$, a mass of $10 M_{\odot}$ for the WR star (with radius $0.92 R_{\odot}$, Langer 1989 ), and $2 M_{\odot}$ and $5 M_{\odot}$ for the compact star (see Table 2). We used 16-s time steps and the number of particles within the escape shell, $30 \times$ separation, was kept constant at $10^{6}$. A new wind particle was always generated when a test particle moved outside the escape shell, returned back to the WR star, or passed closer than $0.15 R_{\odot}$ from the compact star (the assumed accretion radius). In all cases a stable structure was formed after about five binary revolutions.

At large distances (larger than the binary orbit) the simulated outward directional velocity and particle density as a function of 


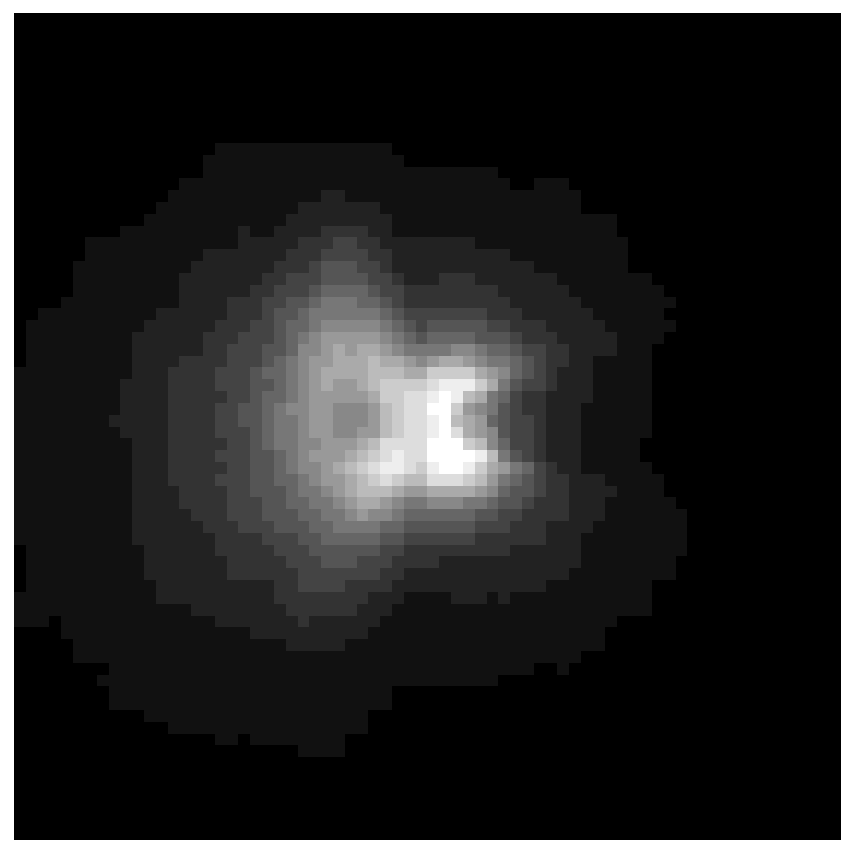

Fig. 4. Si XIV Ly $\alpha$ line emission in the photoionized wind model with $q=5$ and $V=2000 \mathrm{~km} \mathrm{~s}^{-1}$ on the orbital plane. The plot size is $32 R_{\odot}$ (ten binary separations). The grey scale is linear with white corresponding to maximum and black to zero emission. At orbital phases 0.0 and 0.5 the system is viewed from the right and left, respectively.

Table 2. Binary parameters used for wind particle simulations with wind velocities $V=1200-2200 \mathrm{~km} \mathrm{~s}^{-1}$.

\begin{tabular}{lccccc}
\hline \hline $\begin{array}{l}M_{\mathrm{WR}} \\
\left(M_{\odot}\right)\end{array}$ & $\begin{array}{c}M_{\mathrm{C}} \\
\left(M_{\odot}\right)\end{array}$ & $\begin{array}{c}\text { Mass ratio } q \\
\left(M_{\mathrm{WR}} / M_{C}\right)\end{array}$ & $\begin{array}{c}\text { Separation } a \\
\left(R_{\odot}\right)\end{array}$ & $\begin{array}{c}V_{\mathrm{C}} \\
\left(\mathrm{km} \mathrm{s}^{-1}\right)\end{array}$ & $\begin{array}{c}V_{\mathrm{WR}} \\
\left(\mathrm{km} \mathrm{s}^{-1}\right)\end{array}$ \\
\hline 10 & 2 & 5 & 3.2 & 691.0 & 138.2 \\
10 & 5 & 2 & 3.4 & 595.5 & 297.7 \\
\hline
\end{tabular}

the distance, $r$, from the WR component, follow quite closely the semiempirical laws:

$V=$ const. $\left(1-R_{\mathrm{wr}} / r\right)^{\beta} ; N=$ const $/\left[r^{2} \times\left(1-R_{\mathrm{wr}} / r\right)^{\beta}\right] ; \beta=2$.

\section{Line profile fitting results}

\subsection{P Cygni model}

As already remarked in McCollough et al. (2000) and Liedahl et al. (2000) the first HETG spectra of Cyg X-3 showed P Cygnitype line profiles (see also Sako et al. 1999). This is evident also from our phase-resolved Si XIV spectra. We modelled the line profile with two Gaussians, one representing emission (gauss1) and the other absorption (gauss2), as follows:

$F=\exp (-$ gauss 2$) \times($ gauss $1+$ continuum $)$, where

gauss $=\mathrm{A} 0 \times \exp -0.5 \times[(\lambda-\mathrm{A} 1) / \mathrm{A} 2]^{2}$.

The A-parameters are constants obtained in the fitting procedure and $\lambda$ is the wavelength. The continuum was kept constant over the line profile. In practice, $\lambda$ was replaced by the velocity $(\delta \lambda / \lambda \times c)$ measured from the line center. This approach is an approximation of the P Cygni profile. The shape of the feature is a function of the distribution of wind velocities and is more complicated than the simple model above.

The observed profiles were fit using the model described above with the IDL procedure mpcurvefit.pro (written by Craig
Table 3. Radial velocity parameters for the emission component of the Si XIV (6.185 $\AA$ ) and S XVI (4.733 $\AA$ ) Ly $\alpha$ lines and mean $\chi^{2}$-values of P Cygni profile fits.

\begin{tabular}{lccccc}
\hline \hline Line & $\begin{array}{c}\Gamma \\
\mathrm{km} \mathrm{s}^{-1}\end{array}$ & $\begin{array}{c}\mathrm{K} \\
\mathrm{km} \mathrm{s}^{-1}\end{array}$ & $\begin{array}{c}\text { Bluest } \\
\text { phase }\end{array}$ & $\begin{array}{c}\text { MEG } \\
\chi^{2} \text { (d.o.f.) }\end{array}$ & $\begin{array}{c}\text { HEG } \\
\chi^{2} \text { (d.o.f.) }\end{array}$ \\
\hline Si XIV & $64 \pm 38$ & $185 \pm 52$ & $0.52 \pm 0.04$ & $94(26)$ & $62(59)$ \\
S XVI & $75 \pm 33$ & $100 \pm 36$ & $0.44 \pm 0.08$ & $34(26)$ & $40(59)$ \\
\hline
\end{tabular}

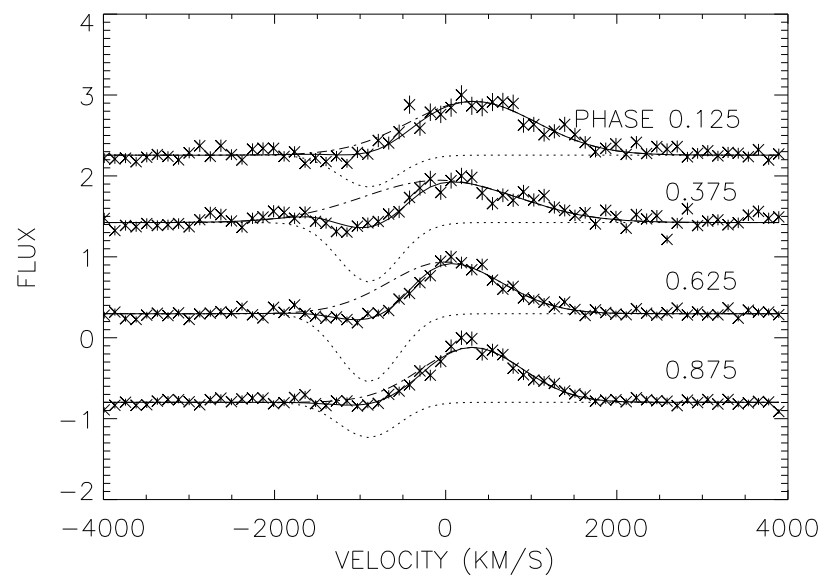

Fig. 5. Si XIV 6.1850 Å Ly $\alpha$ line profiles at four orbital phases (HEG 1st order) overplotted with P Cygni-fits. The profiles are scaled with the maximum value and shifted vertically by a constant amount. The solid line gives the fit, dash-dot line the emission component and the dotted line shows the absorption. The $\mathrm{x}$-axis velocity is measured from the line centrum.

Markwardt). Normal weighting was used (weights $=1 /$ error $^{2}$, where "error" is the observation error of the flux inside a wavelength bin). The line profile was treated within $\pm 4000 \mathrm{~km} \mathrm{~s}^{-1}$ from the line center, corresponding to 66 HEG and 33 MEG $\lambda$-bins at the Si XIV line, respectively. In the final fit the absorption line central velocity was frozen to $-900 \mathrm{~km} \mathrm{~s}^{-1}$, to avoid artificial jumps in some parameters. However, this value is close to what is found when all seven parameters were left free. Mean intrinsic FWHM's of the absorption and emission profiles were $750 \pm 200 \mathrm{~km} \mathrm{~s}^{-1}$ and $1850 \pm 200 \mathrm{~km} \mathrm{~s}^{-1}$, respectively. Due to the requirement of high $\mathrm{S} / \mathrm{N}$, we were restricted to using only four phase bins.

\subsection{Radial velocities}

\subsubsection{The Si XIV line}

The Si XIV Ly $\alpha$ line profile fitting results are shown in Fig. 5 and Table 3. In Fig. 5 the dash-dot line shows the emission component (continuum + gauss1) while the dotted line shows the absorption (continuum - gauss 2 ). In Table 3 we also include the S XVI Ly $\alpha$-line. The $\chi^{2}$ values from the S XVI line fits are somewhat smaller than those of the Si XIV line - this is due to larger observational errors in the latter. Fig. 6 compares the observed Si XIV emission radial velocity curve with the model $(q=5$, $V=2000 \mathrm{~km} \mathrm{~s}^{-1}, i=60 \mathrm{deg}$ ). The rather conservative error bars were computed as errors in the sine curve fit to the mean MEG+HEG data (the solid curve).

The observed radial velocity semiamplitude of the Si XIV emission component was $185 \pm 52 \mathrm{~km} \mathrm{~s}^{-1}$ with a 


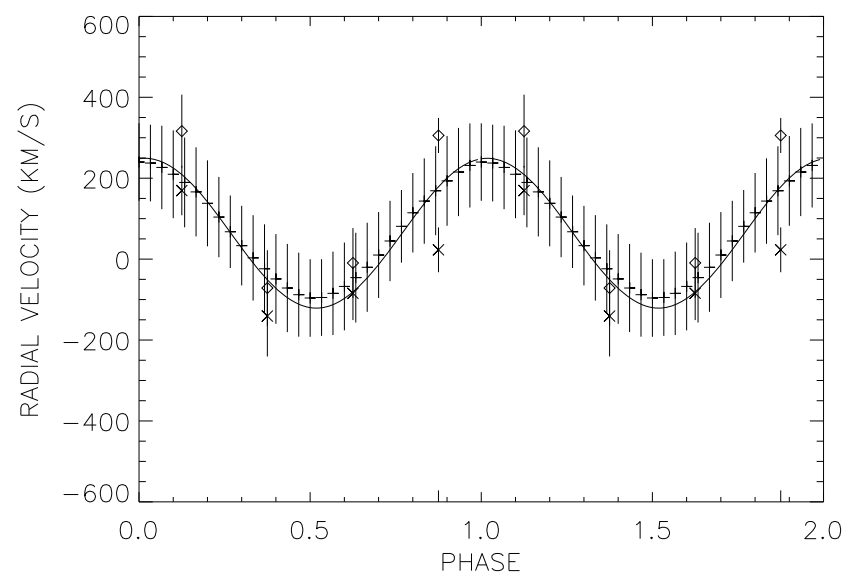

Fig. 6. Radial velocity curve of the Si XIV Ly $\alpha$ emission component at four orbital phases overplotted with the model $q=5$ and $V=$ $2000 \mathrm{~km} \mathrm{~s}^{-1}$ for inclination 60 degrees (dashed line with error bars). The data are represented by symbols with error bars: $M E G=$ crosses, $\mathrm{HEG}=$ diamonds.

Table 4. Effects of inclination, wind velocity and mass ratio on the observed and computed Si XIV radial velocity curves.

\begin{tabular}{lccc}
\hline \hline $\begin{array}{l}\text { Inclination } \\
\text { degrees }\end{array}$ & $\begin{array}{c}V_{\text {wind }} \\
\mathrm{km} \mathrm{s}^{-1}\end{array}$ & $q$ & $\begin{array}{c}\chi^{2} \\
\text { d.o.f. }=28\end{array}$ \\
\hline 60 & 2000 & 5 & 0.84 \\
45 & 2000 & 5 & 3.7 \\
30 & 2000 & 5 & 13.5 \\
60 & 1800 & 5 & 19.9 \\
60 & 2000 & 2 & 6.73 \\
\hline
\end{tabular}

Gamma-velocity of $64 \pm 38 \mathrm{~km} \mathrm{~s}^{-1}$ and maximum blue-shift occurring at phase 0.5 . The S XVI Ly $\alpha$ line at $4.73 \AA$ behaves in a rather similar way but with a somewhat smaller radial velocity amplitude (see Table 3).

The maximum blueshift at phase 0.5 is due to the enhanced emissivity behind the X-ray source (Figs. 3 and 4). To explain the radial velocity amplitude observed, high wind velocity is required. The effects of inclination, wind velocity and mass ratio are compared in Table 4, where $\chi^{2}$-values between the observed and model radial velocity curves are computed for different input parameter values. Due to the conservative errors used, the $\chi^{2}$-values are relatively small. If the mean errors of the observations were to be used, then the $\chi^{2}$ values would be larger by a factor of 5.2.

\subsubsection{The iron lines}

In the Fe-line complex at $6.6-6.9 \mathrm{KeV}$, the dominating $\mathrm{H}-$ and He-type lines are resolved in the HETG-spectrum (see Fig. 2). The lines are formed close to the compact star in a region of high ionizing flux and require special attention. In particular, our ionizing wind models predict a much too weak Fe XXVI line, as can be seen in the large discrepancy between the observed line luminosity and the model (see Table 5). In order to account for this discrepancy, an extra radiation source is required. The He-type Fe XXV line is a composite of forbidden (1.869 $\AA$ ), intercombination (1.859 $\AA$ ) and resonance (1.852 $\AA$ ) lines arising partially in the wind (with intensity ratios $0.35: 0.45: 0.19$ ) and partially in the region where the Fe XXVI line originates.
Table 5. Observed (OBSID 7268 HEG) and model $(q=5, V=$ $2000 \mathrm{~km} \mathrm{~s}^{-1}$ ) line luminosities (in units of $10^{35} \mathrm{erg} / \mathrm{s}$ ).

\begin{tabular}{lccc}
\hline \hline Ion & Wavelength $(\AA)$ & Observed Lum & Model Lum \\
\hline Si XIV & 6.185 & $1.00 \pm 0.08$ & 1.30 \\
S XVI & 4.733 & $1.80 \pm 0.10$ & 1.60 \\
Fe XXV & 1.859 & $7.50 \pm 0.60$ & 2.10 \\
Fe XXVI & 1.780 & $6.05 \pm 0.50$ & 0.90 \\
\hline
\end{tabular}

Table 6. Parameters for the radial velocity curves of Fe XXVI (1.78 $)$ and Fe XXV (1.86 ̊) lines (treated as single Gaussians and using different numbers of phase bins).

\begin{tabular}{lccccc}
\hline \hline Line & Bins & $\begin{array}{c}\Gamma \\
\mathrm{km} \mathrm{s}^{-1}\end{array}$ & $\begin{array}{c}K \\
\mathrm{~km} \mathrm{~s}^{-1}\end{array}$ & $\begin{array}{c}\text { bluest } \\
\text { phase }\end{array}$ & red $\chi^{2}$ (d.o.f.) \\
\hline Fe XXVI & 4 & $28 \pm 95$ & $484 \pm 133$ & $0.27 \pm 0.04$ & $2.85(1)$ \\
Fe XXVI & 10 & $17 \pm 86$ & $454 \pm 108$ & $0.23 \pm 0.04$ & $0.96(7)$ \\
Fe XXVI & 8 & $102 \pm 92$ & $317 \pm 126$ & $0.24 \pm 0.04$ & $0.51(5)$ \\
Fe XXV & 10 & $324 \pm 56$ & $125 \pm 78$ & $0.10 \pm 0.09$ & $0.98(7)$ \\
\hline
\end{tabular}

To study the radial velocity curves we divided the observation into four and ten phase bins. The line centroids and fluxes were determined by single Gaussian fitting in the HEG spectrum. No absorption component was detected, since the spectral resolution is over three times worse than in the Si XIV line region. The wind model predicts for the Fe lines similar radial velocity curves to the cooler Si XIV and S XVI ions, but in fact the observed radial velocities don't correspond to the model. For Fe XXVI, the radial velocity analysis was performed separately for the four and the ten phase bins. In addition, we eliminated the two most blueshifted phase bins and undertook a third study without them (the eight bin case). The results are shown in Fig. 7 and Table 6.

The maximum blueshift of the Fe XXVI line occurs around phase 0.25 , when the compact star is approaching. Hence, it is tempting to associate the line-forming region with the compact component, presumably its accretion disc or corona. If the fit for Fe XXVI is forced to result in a maximum blueshift at exactly phase 0.25 , the resulting $\mathrm{K}$-amplitudes do not deviate significantly from those given in Table 6.

Despite the apparent complexity in producing the Fe XXVI line, we assume that it originates in the vicinity of the compact object, primarily because the maximum blueshift occurs at phase 0.25 when the compact object is approaching.

For Fe XXVI we compromise and adopt the mean $K$ value in Table $6\left(K=418 \pm 123 \mathrm{~km} \mathrm{~s}^{-1}\right)$ to compute the mass function using the definition

$f=K^{3} P /(2 \pi G)=M^{3} \times \sin (i)^{3} / M_{\text {tot }}^{2}$

for a circular orbit. From this we get $f_{\mathrm{WR}}=1.51_{-0.99}^{+1.75} M_{\odot}$. All three cases for Fe XXVI lie, however, within the error ranges. To check this, we also analysed another high state observation of Cyg X-3 (OBSID 6601) that we divided into ten phase bins. The result for FeXXVI was consistent with those of Table 6: $K=$ $470 \pm 130 \mathrm{~km} \mathrm{~s}^{-1}$ when the maximum blueshift was frozen to phase 0.25 (reduced $\chi^{2}=1.3$ ).

The He-like Fe XXV line is more problematic. It is formed partially in the photoionized wind, like Si XIV, and in the vicinity of the compact object, like Fe XXVI. However, extra complications arise due to its triplet nature (resonance, intercombination and forbidden components) which may be selectively absorbed as a function of the orbital phase, and hence we do not treat it further here. 

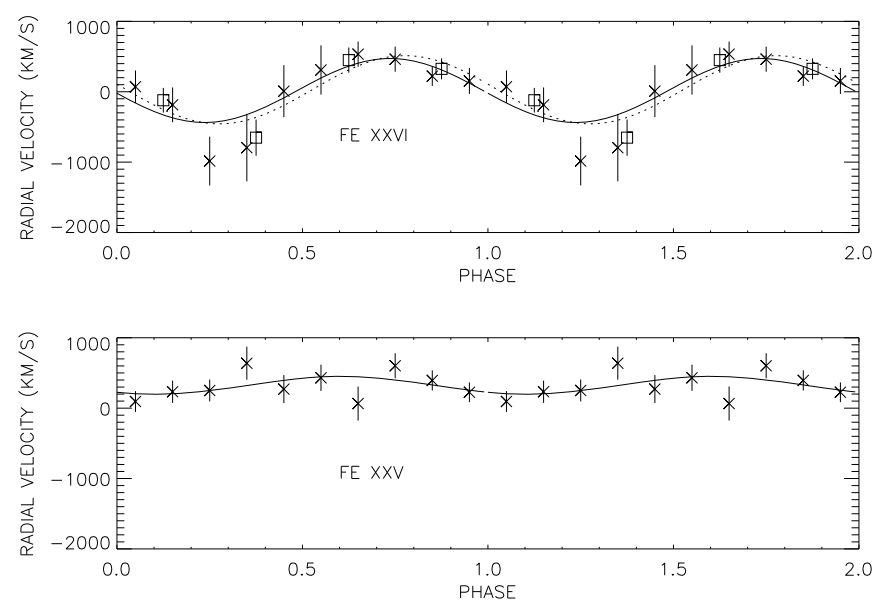

Fig. 7. Upper plot: radial velocity curve of the Fe XXVI (H-like) Ly $\alpha$-line overplotted with the sine curve fit using 10 phase bins (crosses and the solid line). For comparison the same is shown when the observation is divided into 4 phase bins (sqaures and the dotted line). Lower plot: the 10 phase bin case for Fe XXV (He-like). Lines were treated as single Gaussians. Phases 0.0 and 0.5 correspond to the cases when the WR star and compact star, respectively, are in front.

\subsection{Light curves of the emission and absorption components}

In Fig. 8 the integrated area of the Si XIV line absorption component (integrated gauss2) is represented by crosses (from the mean MEG+HEG data) scaled by its maximum value $\left(525 \mathrm{~km} \mathrm{~s}^{-1}\right)$. The continuum (scaled by $5.0 \times 10^{36} \mathrm{erg} / \mathrm{s} / \mathrm{keV}$ ) and the emission component (scaled by $1.5 \times 10^{35} \mathrm{erg} / \mathrm{s}$ ) are represented by diamonds and triangles, respectively.

Approximately one half of the Si XIV line emission originates from the far side of the X-ray source (compact star), accounting for the roughly similar orbital modulation of the line photons and the continuum. The wind particles themselves are relatively symmetrically distributed around the WR component (but asymmetrically around the X-ray component), thus causing the observed orbital modulation via electron scattering and photoelectric absorption. The Si XIV line absorption component is largest around phase 0.5 when the absorbing column is behind the compact star and not penetrating into the higher density wind region around the WR star.

To study the iron lines (where no P Cygni analysis could be used) in the context of the Si XIV line, Fig. 9 was constructed using single Gaussian fits with ten phase bins. As can be seen, the light curves for the lines are rather similar, suggesting the same absorbing agent in all three cases. This might be a combination of continuum opacity for line photons, such as electron scattering, plus broad line absorption probably in the underlying continuum originating from the $\mathrm{X}$-ray source.

\section{Constraints on the system masses and inclination}

Hanson et al. (2000) derive the mass function of the compact star

$f_{C}=0.027 \pm 0.01 M_{\odot}=M_{\mathrm{C}}^{3} \times \sin (i)^{3} /\left(M_{\mathrm{C}}+M_{\mathrm{WR}}\right)^{2}$,

assuming that the infrared He I absorption line at $2.06 \mu \mathrm{m}$ originates in the base of the expanding wind $\left(K=109 \pm 13 \mathrm{~km} \mathrm{~s}^{-1}\right)$ with expected maximum blueshift around phase 0.75 , when the WR star is approaching).

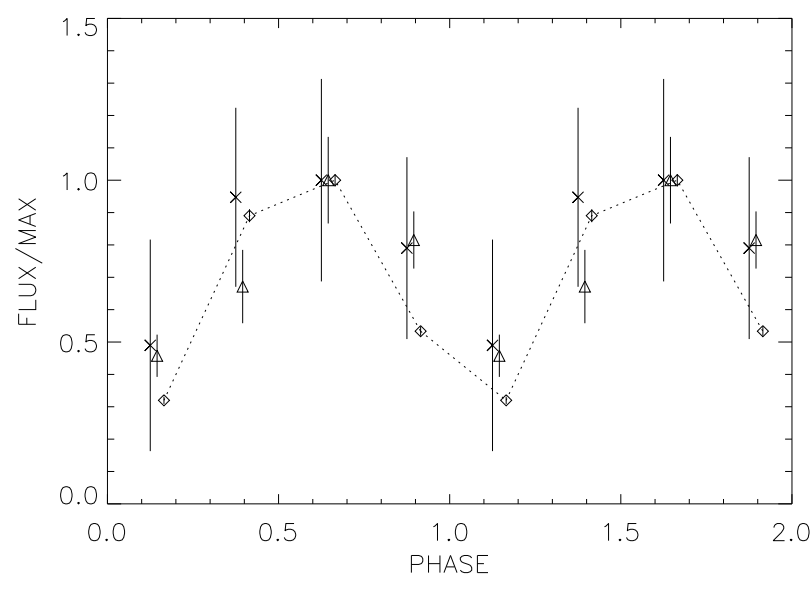

Fig. 8. The integrated area of the Si XIV-line absorption component is represented by crosses, the flux of the emission component by triangles. The diamonds joined by the dotted line gives the continuum at the Si XIV line. All parameters are scaled by their maximum values.
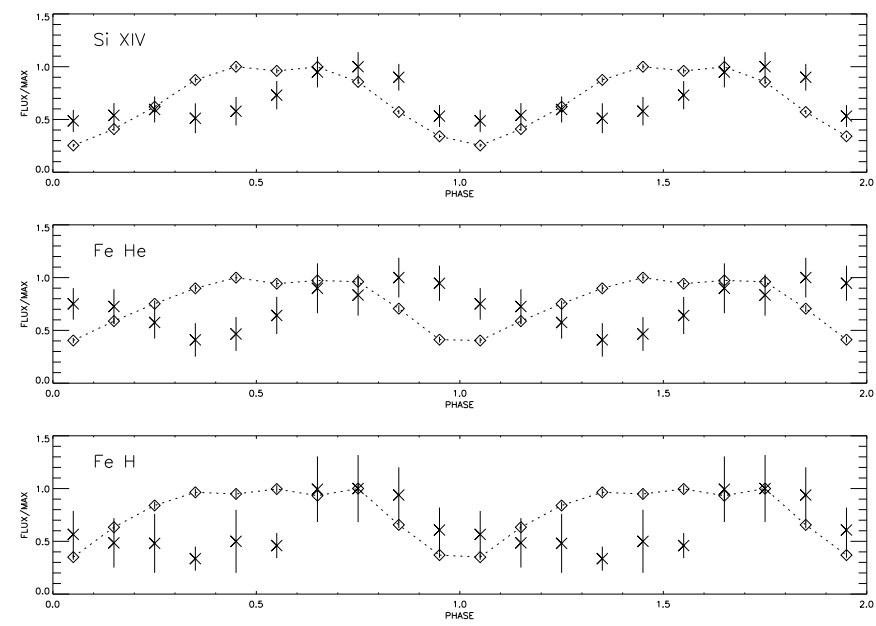

Fig. 9. Scaled continuum fluxes (diamonds joined by dotted lines) and line fluxes (crosses, handled as single Gaussians) as a function of the orbital phase for Si XIV (upper), Fe He-like (middle) and Fe H-like (lower). Phases 0.0 and 0.5 correspond to the cases when the WR star and compact star are in front, respectively.

This mass function can be combined with the mass function we derived from the Fe XXVI line above:

$f_{\mathrm{WR}}=1.51_{-0.98}^{+1.75} M_{\odot}=M_{\mathrm{WR}}^{3} \times \sin (i)^{3} /\left(M_{\mathrm{C}}+M_{\mathrm{WR}}\right)^{2}$.

For the mass ratio we obtain $M_{\mathrm{WR}} / M_{\mathrm{C}}=3.8_{-1.4}^{+1.7}$. Constraints for the compact star mass and inclination are approximately

$M_{C}=2.8_{-1.0}^{+1.2} \times\left(M_{\mathrm{WR}} / 10\right) M_{\odot}$ and

$i=(38 \pm 12) \times\left(M_{\mathrm{WR}} / 10\right)^{0.33}$ degrees.

More mass increases orbital motion; hence a massive system requires smaller inclination to account for the radial velocity amplitude. As an example, if $M_{\mathrm{WR}}=60 M_{\odot}$ then a possible solution (within the error limits) can be $M_{\mathrm{C}}=25 M_{\odot}$ with a small inclination of 15 degrees. This is illustrated in Fig. 10 where the permitted $\left(M_{\mathrm{C}}\right.$, inclination) ranges are shown for three WR masses. 


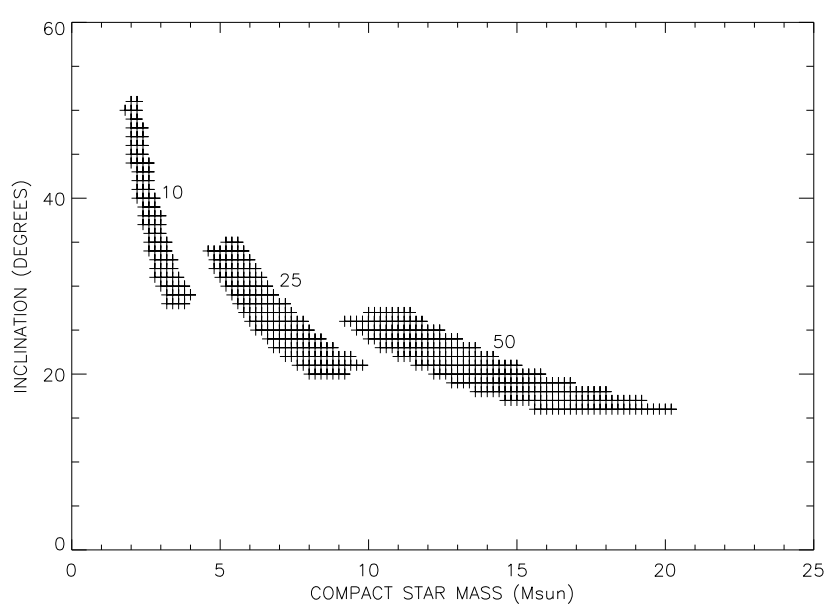

Fig. 10. Permitted (compact star mass, inclination)-ranges for three WR-companion masses marked (in $M_{\odot}$ ) using mass functions from the Fe XXVI (this study) and He I (Hanson et al. 2000) lines.

Inclinations as small as 30 degrees are acceptable for characterizing the amplitude of the Si XIV line. However, the inclination can not be constrained in this way since smaller inclination can be compensated for by larger wind velocity (see Table 4). For large wind velocities the shape of the continuum light curves is not very sensitive on inclination either. The effect of mass ratio on light curves is almost negligible at high wind velocities.

A small inclination is supported by population synthesis results on core He-burning wind-fed $\mathrm{He}+\mathrm{BH}$ binaries by Lommen et al. (2005). They estimate that there is one such system in the Galaxy with an orbital period close to that of Cyg X-3 and satisfying the disc-formation criterion $\left(11 M_{\odot}+5 M_{\odot}\right.$, compare with our Fig. 10). The existence of a disc in Cyg X-3 is supported by the observed superluminal jets. Lommen et al. (2005) consider unlikely the existence of similar WR+NS binaries with discs, as already shown by Ergma and Yungelson (1998), due to the large wind velocity and small angular momentum of the $\mathrm{WR}+\mathrm{NS}$ wind that hinder disc formation. On the other hand, Martí et al. (2001) detected a bipolar jet flow at a large angle (73 degrees) to the line of sight. If the flow is parallel to the orbital plane axis, then this favours large inclination. However, this need not be the case for a wind-disc scenario as discussed by Martí et al. (2001) who assume an inclination of 30 degrees (see their Fig. 7). If this is indeed the case, then small inclination is favoured.

Gladstone et al. (2007) consider a black hole signature to be a source penetrating into the ultrasoft domain in their colourcolour diagram (where Cyg X-3 never entered). However, if the mass of the black hole is small, then its disc is hot, keeping it out of the ultrasoft domain populated by more massive black holes. On the other hand, based on spectral evolution vs. the Eddington luminosity, Hjalmarsdotter et al. (2008b) point to a massive $\left(30 M_{\odot}\right)$ black hole, and consequently a very small inclination, with a massive WR companion.

Support for low inclination in our photoionized wind model comes from the infrared. We computed the emissivity and radial velocities of the IR He II $2.09 \mu \mathrm{m}$ emission line (using atomic data provided by T. Kallman, private communication). It turns out that most of the line emission comes from the X-ray shadow (see Fig. 3) where the WR star is the only ionizer. The radial velocity $\mathrm{K}$-amplitude is around $500 \mathrm{~km} \mathrm{~s}^{-1}$ with maximum blueshift at phase 0.0, i.e. 180 degrees from that of the Si XIV

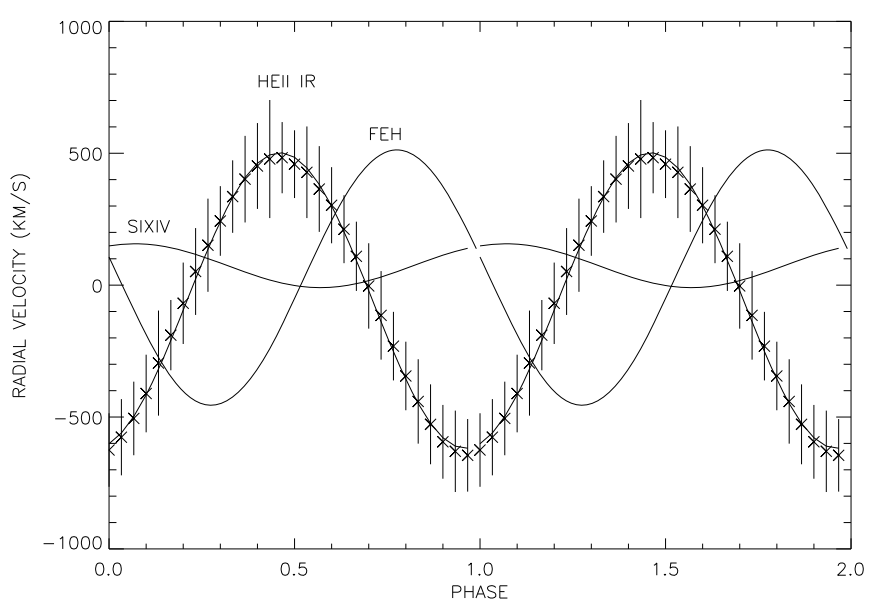

Fig. 11. Model $\left(q=5, V=2000 \mathrm{~km} \mathrm{~s}^{-1}\right)$ radial velocity curve of the infrared He II $2.09 \mu \mathrm{m}$ line for inclination 30 degrees. The line is formed in the X-ray shadow cone behind the WR component where the wind is photoionized by the EUV radiation from the WR star. The fits for the observed Si XIV and Fe XXVI (H like) lines are shown for comparison. These lines are formed in the X-ray photoionized wind and at the compact star, respectively.

emission, as observed by Hanson et al. (2000). However, in our model with a wind velocity of $2000 \mathrm{~km} \mathrm{~s}^{-1}, q=5$ and inclination 60 degrees, the computed $\mathrm{K}$ value is much larger than that observed $\left(1020 \mathrm{~km} \mathrm{~s}^{-1}\right.$ with maximum blueshift at phase 0.0$)$. If the inclination is 30 degrees, the resulting amplitude of $580 \mathrm{~km} \mathrm{~s}^{-1}$ would be closer to that observed (see Fig. 11) and the permitted ranges of the WR and compact star masses are 10-40 $M_{\odot}$ and 2.8-8 $M_{\odot}$, respectively. For an inclination of 60 degrees the same ranges are 5-8 $M_{\odot}$ and 1.0-3.2 $M_{\odot}$.

\section{Discussion}

Our mass estimates (see Fig. 10) corroborate the Hanson et al. (2000) conclusion that if the compact component is a neutron star with inclination greater than 60 degrees, then the WR mass must be in the range 5-11 $M_{\odot}$. Stark and Saia (2003) gave an upper limit of $3.6 M_{\odot}$ for the compact star mass using the Helike Fe XXV line with a similar radial velocity curve to ours (see Fig. 7) - however, we prefer to use the H-like Fe XXVI line here and defer a more in-depth discussion of the He-like Fe XXV line to a separate paper. They also observed radial velocity curves of the Si XIV and S XVI lines very similar to ours. The difference is in their larger Gamma (recession) velocities which are due to ignoring the P Cygni absorption and treating the lines as pure Gaussians.

The formation mechanism of the Fe XXVI line at $1.78 \AA$ remains unknown, although it was proposed above to arise in the vicinity of the compact object. If the line is formed in a hot collisionally dominated plasma with temperature $T=10 \mathrm{KeV}$, the required emission measure $\mathrm{EM}=n^{2} \mathrm{~V}$ is around $10^{60} \mathrm{~cm}^{-3}$ (for a distance of $9 \mathrm{kpc}$ ) using the MEKAL model in XSPEC. The emitting plasma can be e.g. a spherical corona with radius $10^{10} \mathrm{~cm}$ (roughly the assumed accretion radius) and electron density $5 \times 10^{14} \mathrm{~cm}^{-3}$. The corona itself can also be a composite of many impulsive flares. Another alternative is that the hard coronal flux is photoionizing the disc providing additional Fe emission. 
In our particle simulations we assumed $M_{\mathrm{WR}}=10 M_{\odot}$ and one may ask how a larger WR mass would influence the model results. A larger mass increases the separation and, hence, lowers the wind density in line forming regions. Keeping all other parameters fixed, the major effect of this would be the decreasing of model line luminosities in Table 5. Unfortunately, the masses of WR stars have a large spread even for the same spectral type (see Fig. 4 of Crowther 2007).

The question of the origin of line absorption (Sect. 5.3) is interesting, even though not fully solved here. The similarity of the Si XIV, Fe XXV and Fe XXVI light curves (Fig. 9) is indicative of the same absorption agent for all. The P Cygni treatment of Si XIV (Fig. 8) leads us to speculate that line photons are absorbed in the same fashion as the continuum. In addition, there can be line absorption operating in the continuum (below the line photons) that is blueshifted and varies with the orbital phase. A combination of these two absorptions might then cause the broad minima seen in Fig. 9. We leave a deeper discussion on line absorption for a separate paper.

\section{Conclusions}

We have shown that the Si XIV (6.185 $\AA$ ) and S XVI (4.73 $\AA$ ) Ly $\alpha$ emission lines of Cyg X-3, using Chandra HETG observations, have P Cygni-type orbitally modulated line profiles. The emission components arise in the wind of the WR-companion, photoionized and excited by a hybrid source, which we take to be composed of the compact X-ray source and WR He-star.

The radial velocity curves of the emission components have maximum blue-shifts at phase 0.5 , when the compact star is in front, as predicted by the wind model. The K-amplitudes depend mostly on the wind velocity and constraints on the mass ratio and orbital inclination are weak. The absorption component velocities are constant around $-900 \mathrm{~km} \mathrm{~s}^{-1}$. Both the absorption and the emission components have intensity maxima (integrated Gaussians) around phase 0.5 , similar to the continuum flux.

The Fe XXVI Ly $\alpha$ line at $1.78 \AA$ appears to arise in the vicinity of the compact star, as maximum blue shift occurs at phase 0.25 which is when the compact star is approaching. Combining the mass function derived from this line with the mass function from the IR He I absorption line (Hanson et al. 2000) we were able to constrain the system masses and inclination. Our photoionization simulations of the IR He II $2.09 \mu \mathrm{m}$ emission line (formed in the X-ray shadow) lend support to a small orbital inclination (30 degrees) for which the range of masses of the compact star is $2.8-8.0 M_{\odot}$ For a larger inclination of 60 degrees this range would be $1.0-3.2 M_{\odot}$.
In the light of our results, we either have a neutron star and low-mass Wolf-Rayet star in a system of large orbital inclination or a black hole whose mass depends on the Wolf-Rayet mass and the system orbital inclination.

Acknowledgements. We thank Dr. Tim Kallman for advice in using the XSTAR code and providing additional IR line atomic data. D.C.H. is grateful to the Academy of Finland for a Fellowship. We thank the anonymous referee for pushing us to improve the presentation. This research has made use of data obtained from the Chandra Data Archive and software provided by the Chandra X-ray Center (CXC) in the application packages CIAO, ChIPS, and Sherpa.

\section{References}

Cox, A. N. (ed.) 2000, Allen's Astrophysical Quantities, Fourth Edition (New York, Berlin, Heidelberg: AIP Press, Springer-Verlag)

Crowther P. A., Smith L. J., \& Hillier, D. J. 1995, Hot Star Newsletter, 6, 28 (Feb. 1995)

Crowther, P. A. 2007, ARA\&A, 45, 177

Ergma, E., \& Yungelson, L. 1998, A\&A, 333, 151

Fender, R. P., Bell Burnell, S. J., Williams, P. M., \& Webster, A. S. 1996, MNRAS, 283, 798

Fender, R. P., Hanson, M. M., \& Pooley, G. G. 1999, MNRAS, 308, 473

Gladstone, J., Done, C., \& Gierlinski, M. 2006, MNRAS, 378, 13

Hanson, M. M., Still, M. D., \& Fender, R. P. 2000, ApJ, 541, 308.

Hjalmarsdotter, L., Zdziarski, A. A., Larsson, S., et al. 2008a, MNRAS, 384, 278

Hjalmarsdotter, L., Zdziarski, A. A., Szostek, A., \& Hannikainen, D. 2008b, MNRAS, submitted

Kallman, T. R. 2006, A Spectral Analysis Tool, version 2.1kn6, Goddard Space Flight Center, May25, 2006

van Kerkwijk, M. H., Charles, P. A., Geballe, T. R., et al. 1992, Nature, 355, 703 van Kerkwijk, M. H., Geballe, T. R., King, D. L., et al. 1996, A\&A, 314, 521

Langer, N. 1989, A\&A, 210, 93

Liedahl, D. A., Sako, M., Paerels, F., et al. 2000, AAS HEAD Meeting, BAAS, 32,1220

Liedahl, D. A., Wojdowski, P. S., Jimenez-Garate, M. A., \& Sako, M. 2001, Xray Spectroscopy of Accretion Disks and Stellar Winds in X-ray Binaries, ASPC, 247, 417

Liu, Q. Z., van Paradijs, J., \& van den Heuvel, E. P. J. 2007, A\&A, 469, 807

Lommen, D., Yungelson, L., van den Heuvel, E., Nelemans, G., \& Zwart, P. 2005, A\&A, 443, 231

Martí, J., Paredes, J. M., \& Peracaula, M. 2001, A\&A, 375, 476

McCollough, M. L., Elsner, R. F., Dieters, S., et al. 2000, AAS HEAD Meeting, BAAS, 32, 1228

Paerels, F., Cottam, J., Sako, M., et al. 2000, ApJ, 533, L135

Poutanen, J., \& Svensson, R. 1996, 470, 249

Pringle, J. E. 1974, Nature, 247, 21

Sako, M., Liedahl, D. A., Kahn, S. M., \& Paerels, F. 1999, ApJ, 525, 921

Sako, M., Kahn, S. M., Paerels, F., et al. 2002, in High Resolution Spectroscopy with XMM-Newton and Chandra, invited review, MSSL workshop Oct. 24-25 [arXiv: astro-ph/0309503]

Stark, M. J., \& Saia, M. 2003, ApJ, 587, L101

Szostek, A., \& Zdziarski A. A. 2008, MNRAS, in press, [arXiv:0710 . 5591]

Vilhu, O., Hjalmarsdotter, L., Zdziarski, A. A., et al. 2003, A\&A, 411, L405

Vilhu, O., Hakala, P., Hjalmarsdotter, N., et al. 2007, in The Obscured Univerese, ed. S. Grebenev, R. Sunyaev, \& C. Winkler, ESA SP-622, 377

Willingale, R., King, A. R., \& Pounds, K. A. 1985, MNRAS, 215, 295 